\title{
SOME POSSIBLE CAUSES OF CONGENITAL HEART MALFORMATION
}

\author{
BY \\ G. E. H. FOXON \\ From the Department of Biology, Medical School of Guy's Hospital
}

Received April 30, 1958

Certain conditions found in human congenital heart disease are probably genetic in origin (Cockayne, 1938; Polani and Campbell, 1955), but sometimes they may be correlated with an environmental factor, namely the infection of the mother with rubella during early pregnancy. Thus there appears to be no single cause of congenital heart abnormality; sometimes genetic causes and sometimes environmental causes are implicated. The purpose of this article is to draw attention to certain general problems of heart development in vertebrate animals and to some recent experimental work on various vertebrates which may help towards a clearer understanding of the parts played by genetic and environmental factors in the production of congenital abnormalities of the heart.

\section{Genetic and EnVironmental Factors in Heart Development}

When a zygote is formed by the union of gametes, it will develop into a normal animal if its genetic constitution is normal and if the environment in which development takes place is normal. The difficulty that arises in considering all forms of abnormal development is to distinguish between abnormalities due to genetic factors and those due to environmental factors. In the case of human abnormalities it is desirable to ascertain the cause of abnormality if, by so doing, it is possible to take any action that will lessen the likelihood of the birth of congenitally malformed children. If, for example, it could be shown that hereditary factors were involved, advice on the desirability or otherwise of marriage between certain partners could be given. If environmental factors were shown to be implicated, it might be possible to prevent such conditions arising by ante-natal care. Clearly in congenital heart disease the causes of abnormality are of more than academic interest. The difficulty of separating genetic and environmental causes arises from the fact that, whatever the cause, the abnormality is only recognizable after it has been formed, that is when both hereditary and environmental factors have made their contribution to the form of the organ under consideration.

The early formation of an organ is clearly under genetic control. The cells that occupy a certain position in an embryo normally form certain tissues and organs of that embryo, and experimental analysis of embryos has shown when in time the fate of cells in certain parts of the embryo becomes fixed, and also how certain cell masses, as well as becoming fixed in their ultimate fate, have the power of inducing neighbouring cells to take on a certain form of development in their turn. This is equivalent to saying that the fate of a cell is determined in relation to its position with regard to other cells or to its cellular environment. The differences between cells at this stage are chemical ones and they are said to be chemo-differentiated, whereas when visible differences have been established they show histo-differentiation. Such chemo-differentiation is due to the interplay of the genetic constituents of the nucleus and the cytoplasm which forms its environment. This cellular environment is under genetic control and the factors operating here are genetic ones. This phase of development quickly merges into another in which internal mechanical forces, set up when the heart begins to function, clearly form an important part of the internal environment. These forces will fall to be 
considered in some detail, as several authors have attributed abnormal heart development largely to abnormal mechanical stresses at this stage. Clearly such abnormal stresses could be brought about by previous genetic variations altering the normal course of blood flow, in which instance such abnormality would be genetic in origin. Or it might be that some factor external to the embryo might interfere with either the speed or volume of the blood flow in which instance the abnormality would be attributable to factors in the external environment.

External environmental factors that are clearly not under genetic control are those that have to do with the supply of nutrients, gases, and other materials needed by the developing embryo.

Finally, it is necessary to mention the influence of radiation and other external factors, such as mitotic poisons, which may have an influence on the developing embryo through its genetic constitution and not by simple mechanical interference during the course of morphogenesis.

\section{General Anatomical Features of Heart Development}

The heart in all vertebrates is formed from two mesodermal primordia, left and right, which unite below the midline of the gut. In the developing chick, before union, these primordia take the form of endocardial tubes, and they first coalesce in the region that will form the bulbus cordis, their further union taking place in a caudal direction. The single tube of endothelium thus formed is quickly covered by the myocardial layer and, as soon as this has happened, the twitchings in this musculature mark the beginning of the heart's activities.

As is well known, the heart does not remain as a straight tube but takes on the typical Sbend whereby the atrial region comes to lie cephalad of the ventricular region, and also the twisting is such that the ventricle comes to lie distinctly to the right of the midline. In those hearts in which the left and right sides become separated, i.e. in mammals and birds, subsequent changes of disposition have to take place to correct this extreme right movement of the ventricular portion, so that longitudinal partitions dividing the atrium, the ventricle, and the bulbus cordis come to lie in approximately the same straight line. The details of the processes by which this is accomplished in birds and in mammals are not the same; this is only to be expected in view of the separate origins of these groups from early reptiles, which will be mentioned later.

Hand in hand with the development of the heart, itself, goes the development of the six pairs of arterial arches (Fig. 1). In all the land-living vertebrates the first two pairs make but brief appearances and are already declining before the more posterior vessels are fully formed. The third pair of arches, which give rise to the carotid arteries, are well developed in both birds and mammals and in both these groups the fourth pair also appear. In birds (Fig. 1, D) the fourth right arch becomes the definitive aortic arch, and into this arch opens the ductus arteriosus of the right side. The fourth left systemic arch in birds disappears but, nevertheless, there is a ductus arteriosus on the left side as well, which joins the sixth arch to what appears to be the remains of the left systemic but is really part of the lateral dorsal aorta of that side. In mammals (Fig. 1, E) both fourth arches persist, the left as the root of the aorta joining the lateral dorsal aorta, the right as the base of the subclavian artery, the right lateral dorsal aorta disappearing in this case. In both birds and mammals the sixth arches form the pulmonary arches. The fifth pair of arches are usually regarded as being evanescent in their appearance but, in work that will be reviewed below, Stephan (1952) reported that it frequently did not appear in embryo chicks and it seems equally difficult to find in embryo mammals.

Abbreviations used in Fig. 1 and 2.

1-6 The six aortic arches

an. anastomosis

d.c. ductus caroticus, patent in some reptiles

l.s.a left systemic arch

1.s.c. left subclavian artery

p.a. pulmonary artery

r.du.a. right ductus arteriosus

r.s.a. right systemic arch

s.s.c. secondary subclavian (of crocodiles and birds) x. the external carotid artery becomes interrupted in this region 

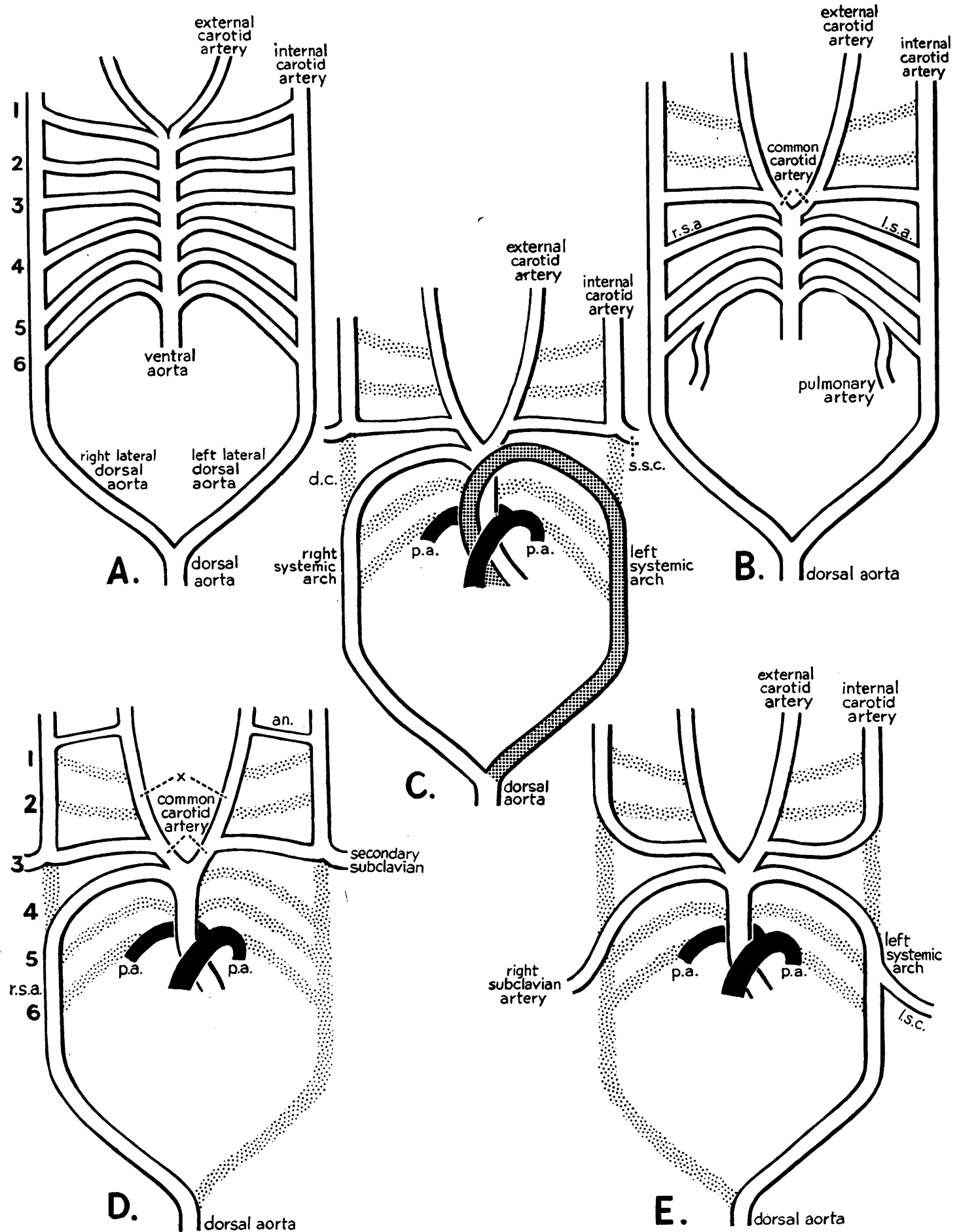

FIG. 1.-The normal fate of the primary six pairs of arches, ventral view. (A) Embryonic stage, all six arches shown. $\begin{array}{llll}\text { (B) Urodele amphibian. (C). Reptile. (D) Bird. (E) Mammal. The arches that disappear in development are } & \text { (D) }\end{array}$ stippled; venous blood, black; mixed blood, shaded. All vessels are shown in an arbitrary manner related to the primitive scheme; in life they are generally straightened out and their proportions altered. In birds (D) a secondary anastomosis (an.) develops between internal and external carotid at the top of the neck, and external carotid disappears at $x$. The secondary subclavian is a character shared by birds and crocodiles. 


\section{Phylogeny and Heart Development}

Several authors, but notably Spitzer, have drawn attention to the fact that many anomalies of the heart and arterial arches in man are highly reminiscent of the definitive arrangement of the heart and great vessels in the adults of various reptiles and amphibians. Such conditions, it has been suggested, represent the anatomical arrangement that obtained in vertebrates ancestral to mammals, which by a process of arrest of development is retained as the final state.

It seems necessary therefore to draw attention to the present position of the recapitulation theory. This theory has been critically restated in recent years by Garstang (1921) and by de Beer (1951). The older idea of this theory was often summarized in the phrase "ontogeny repeats phylogeny" or, to put it more crudely, that an animal climbed up its genealogical tree. It is now realized that animals do not evolve merely by prolonging their development and adding new characters at the end of their developmental stages, but by a gradual modification of the whole course of development so that the end result is not precisely the same as the animals' more remote ancestors. To put this another way, just as the final form of an animal undergoes evolutionary change, so its developmental stages undergo evolutionary change: thus the developmental stages of the animal are not to be looked upon as anything more than a vague reminiscence of the embryological stages of its ancestors. It is now generally supposed that such likenesses, as, for example, that shown between the existence of a double aortic arch in man and the similar condition displayed in the frog, are to be explained by the well known fact that, during the development of all vertebrates, six pairs of aortic arches normally appear and that in some vertebrates certain of the arches disappear and in other vertebrates other arches disappear. Where arches that normally disappear persist, the fact that they may then resemble those of another group of vertebrates, in which such arches are normally kept, gives rise to a resemblance that is only remotely the result of phylogeny. Thus a man with a double aortic arch has this because development proceeded incorrectly and not because some very remote ancestors of man were "amphibians."

The time factor is also of importance. It must be remembered that, since the amphibians gave rise to early reptiles some 250 million years ago, the amphibians have themselves undergone extensive evolutionary change, and the structure of these amphibians was not always the same as that of amphibians of today. In the skeletal elements where those of living and extinct forms may be compared, many remarkable differences are demonstrable.

Another matter of phylogenetic importance is that, although both birds and mammals have fourchambered hearts, it is now realized that this similarity has been brought about by the evolutionary development of hearts with the left and right side perfectly separated on two distinct occasions in vertebrate history. This is not the place to survey the evidence on which this statement is based; the idea is primarily due to Goodrich (1916). The evidence for this view which has been summarized elsewhere (Foxon, 1955) has to do with (1) the incipient division of the ventricle in living reptiles and the relations of these divisions with the two systemic arches, (2) the relations of the carotid arteries with the systemic vessels, and (3) differences in the mode of embryological development of the ventricular septum in reptiles, birds, and mammals. Consideration of these points has led to the belief that after vertebrates had emerged as land-living animals in late Devonian times it was not long, at least on the geological time-scale, before in the Carboniferous times reptiles arose and divided into two groups. Fossil reptiles can be assigned to one or other group by skeletal characters. It is thought that in one group evolution proceeded so that the oxygenated blood from the left side of the ventricle was delivered into the left systemic arch and that with this arch the carotid vessels became associated. This group of reptiles gave rise to the mammals but left no other descendents. In the other group of reptiles (Fig.1C) the blood from the left side of the heart was delivered mainly into the right systemic arch with which the carotid arteries became associated; and the left systemic arch carried more venous blood. This type of circulation persists in the reptiles that exist in the world today and also in the birds, where, however, the left systemic arch does not persist beyond the embryonic stage. 
From the foregoing remarks it will be seen that, although phylogenetically there is a wide gulf between the mammalian heart and the avian heart, there are many principles of heart development that apply to the hearts of all vertebrates, and it would seem reasonable to use evidence obtained by the study of the avian heart in arguments concerning the mammalian heart, so long as they deal with the general principles of development and not with those details that must belong either to the one line of evolution or to the other.

\section{Organization of Heart Development}

The heart rudiment is formed from mesoderm that is quite early determined as potential heart tissue, and the location and extent of this tissue has been mapped for the chick by Rudnick (1944). The fact that two laterally situated rudiments take part in this formation, forming two endothelial tubes, is liable to be misunderstood in the case of the higher vertebrates. These two tubes have nothing to do with the division of the heart into left and right sides in mammals and birds; it is true that it is possible in certain cases to follow the contribution made by each of these rudiments to the definitive heart, as has been done by Pernkopf and Wirtinger (1933), but in amphibians, where the heart is similarly formed from two rudiments, it is known that the rudiment of each side, if it fails to join with its fellow, is capable of forming a complete heart rudiment itself, or, if the joint rudiment is divided longitudinally into three, three hearts will begin to form. The ability to form a complete heart, however, is not equally present in both sides of the combined rudiment but seems to predominate on the left. Conversely, if, at the neurular stage of development of the embryo, two heart rudiments are grafted together, they can regulate to form one normal heart provided that the antero-posterior axes are similarly orientated (for references to this work see summary in Huxley and de Beer, 1934). These facts are of course well known, but more recently Fales (1946), in studying the heart of Amblystoma punctatum, has drawn attention to the fact that hearts formed from right rudiments usually show reversed symmetry, being mirror images of left hearts, although this is not absolute for all amphibian hearts, as Ekman (1925) had found in Bombinator. Fales found that, while hearts from the left and right rudiments were apparently capable of developing in an essentially normal manner, they did not necessarily do so, and this was apparently caused by the pressure of the surrounding structures; she suggested that the reversal of symmetry exhibited by right hearts was caused by pressure, for, if the prospective left heart tissue was removed, the right tissue produced a normal heart. It would seem most likely, however, that the initial asymmetry exhibited by all vertebrates, shown not only by the heart but by the asymmetrical development of the alimentary canal as well, is of genetic origin. Huxley and de Beer say that this asymmetry factor is situated in the gut roof for, if a portion of the gut roof in the region of the presumptive heart tissue is reversed through $180^{\circ}$ when the developing embryo is at the open neural groove stage, reversal of the symmetry of the heart is brought about.

The suggestion of Fales (1946) on the influence of pressure of surrounding tissues mentioned above draws attention to the mechanical influence of such surrounding tissue. By many authors this has been considered to play an all important part in heart development.

\section{Mechanical Factors in Heart Development}

Mechanical factors must be distinguished, if it is possible, from external environmental factors. In the normal development of an animal, as has been mentioned, all parts of the organism exert influences on neighbouring parts. At first such influences are largely to be described in terms of the working out of the gene complex by chemical means under such headings as differentiation and induction, but later organs exert simpler forces on each other, such as mechanical processes, and seem to push and pull on each other and, as already mentioned, the blood flow itself exerts such pressures. In normal development such pressures must be regarded as normal and are the result of growth and 
differentiation that have gone on previously. In other words, the mechanical factors are in their turn a working out of the hereditary factors of the developing embryo.

If it can be shown that the mechanical factors become abnormal in some respect, it becomes necessary to enquire whether their abnormality is to be ascribed to abnormal genetic effects at an earlier stage or to some unusual factor in the environment of the embryo itself which can be described as an external environment cause.

Some authors have attributed a large amount of the asymmetrical twisting and bending seen in the early stages of heart development to the presence within the heart of moving streams of blood. Spitzer placed great emphasis on blood flow as a factor in heart development. Bremer too (1928, 1932), has devoted much time to the elucidation of the influence of the blood passing through the heart on heart formation. Some ideas have been admittedly rather crude and liken the streams of blood flowing through the heart to the erosive forces of currents of water flowing in a river, whereby, if blood flows strongly it obtains a free passage, and where its movement is sluggish endothelial proliferations may form obstructions, which in the higher vertebrates take the form of the heart septa. Although these streams of blood are clearly demonstrable, as has been shown by Bremer and also by Goerttler (1955), heart development, as the development of all other organs, must be a mixture of hereditary factors and the factors imposed in the immediate environment, of which of course, the blood stream is one.

Turning to consider the effect of blood on the development of the heart in more detail, it should be noted that Fales, working on Amblystoma, concluded that there was no evidence to indicate that the external shape of the heart depended on the presence of flowing blood. Hearts in which very little blood flow was taking place nevertheless had a normal shape, but Fales concluded that flowing blood was necessary for the hollowing out effect, for hearts without blood frequently had thicker walls, particularly in the ventricle, and the conus might be partially solid or only have a very narrow cavity. Bremer, working with chick embryos, found that from an early stage there were two streams of blood entering the heart from the vitelline veins which coursed around each other in spiral fashion. In the ventricle and conus regions, the region of stiller blood between the two streams was shown to be the line of future ventricular and bulbar septa; the interatrial septum, however, developed across one of the streams of blood in the atrium. Later one stream was taken over by the pulmonary return and the other by the venous return. This would show that the interatrial septum at any rate develops without the stimulus of blood flowing along its two sides. Simons (1957) has shown that in frog tadpoles, in which the removal of the lung rudiments had ensured that there was no pulmonary circulation, the atrial septum developed normally, although it did not separate two streams of blood. The actual size, however, of the left division of the atrium was much reduced and therefore the normal flow of blood is necessary for this cavity to attain its normal proportions but not for the actual development of the septum.

The experiments just quoted seem to show that in the atrial region of the heart at least there is a considerable genetic factor at work irrespective of mechanical factors: that is, in animals in which an interatrial septum normally develops, it will develop whether the blood flow is normal or not. This, it would seem, is an argument for the view that each particular species of vertebrate will possess hereditary factors that, during morphogenesis, will guide the development of the heart and arterial system so as to produce ultimately a pattern of heart and arterial structure characteristic of the species.

Rather cruder mechanical reasons have, however, been suggested by some authors. For example Bremer endeavoured to show that the left systemic arch of the chick underwent regression because, when the heart reached that stage of development in which it moves back into the thoracic region, the heart also became twisted in relation to the origin of the two systemic arches hitherto symmetrical. This twisting, or rather untwisting, of the original heart flexure, as Bremer claimed it to be, had the result that the orifice of the right systemic artery was brought into the position of receiving the bulk of blood flowing through the arterial division of the bulbus, whereas the left systemic received only a slight flow. According to Bremer, when the left vessel only received a slight flow, changes took 
place, such as proliferation of the endothelium, that had as their result the complete occlusion of this vessel. Stephan (1952), who has made a study of the fate of the arterial arches in the chick, indeed found that the normal method of closure was for endothelial proliferation to take place and for a gradual slowing up of the blood stream and its final cessation. Such evidence in conjunction with that of Fales already quoted, who found that in amphibian hearts which gradually ceased to beat similar proliferation took place with cessation of circulation, thus suggests that mechanical factors play a large part in these processes. However, it seems a large step from saying (1) that in the disappearance of the left systemic arch of the chick certain phenomena of mechanics and growth are seen which bring about the end result, to saying (2) that the end result is brought about by a number of mechanical processes following each other automatically and that genetic control, itself, ceases when the asymmetry factor ceases to operate.

Further correlation between genetic and mechanical factors can be seen in the differentiation of the other parts of the heart and the arterial vessels. As has been explained before, all the vertebrate blood systems can be related to a hypothetical scheme, presumably present in some remote ancestor, in which there were six pairs of arterial arches present anterior to the heart, and the fifth pair appears very rarely and then only fleetingly in the higher vertebrates (not at all in the rat). It might be assumed that, if its disappearance is a matter of mechanics and not genetics, this fifth pair or one of the pair could perhaps be encouraged to develop or persist in embryos in which the other vessels of one side were occluded, thus forcing more blood through the rudiment of the fifth arch. Such experiments have in fact been made by Stephan (1952).

Stephan, working on chick embryos of four days' incubation, devised a method whereby he could ligature one or more of the arterial arches. These chicks continued with their development until a late stage and some even hatched. By this means he produced a wide variety of arterial arch patterns, the most bizarre arrangements being sometimes produced, e.g. Fig. 2, C and D. Except in a few rare instances where peculiar anastomoses took place, these patterns could be explained by the persistence of parts of the third, fourth, and sixth pairs of arches, together with the lateral dorsal aortæ. He did not succeed in forcing parts of the first, second, or fifth arches to remain. It seems, therefore, that the disappearance of the first, second, and fifth arches is not entirely a matter of simple mechanics but that some genetic factor is involved. On the other hand, the lateral dorsal aorta of both sides readily persisted as a mechanical necessity (Fig. 2, C and D).

In instances in which both sixth arches were suppressed, the pulmonary arteries obtained a modified form of blood supply, but of course the obliteration of the pulmonary trunk means that, if the ventricle were to be completely divided, the right side would have no outlet. Stephan indeed found that gross abnormalities of the arterial arch pattern, such as those just quoted, had an influence on the completion of the ventricular septum and, in fact, the septum remained incomplete in those instances where its completion would have made the circulation impossible. These experiments may therefore throw some light on interventricular septal defects.

Finally, in this section on mechanical factors, attention must be directed to the work of Shaner (1949) on pig embryos. He thought that some defect at an early stage in the atrio-ventricular canal deflected the blood streams in an abnormal way as they passed through into the ventricular cavity. This abnormal development of endothelial cushions in the A-V canal reduced blood flow on one or other side of the developing bulbus. This usually resulted in pulmonary stenosis, the aortic root being not so likely to be affected. There is no suggestion, however, as to what caused the primary disturbance of the cushions in the A-V canal, but it does emphasize the importance of an abnormality in one part of the heart affecting another region at a later stage.

\section{The EXTERNAL ENVIRONMENT OF THE EMBryo}

In the preceding section of this paper attention has been drawn to the fact that certain experimental interference with the embryo will upset the normal course of development of the heart and arterial system. Experimental modification of the blood flow shows how such abnormal blood flow can influence the further development of the heart and vessels. What has not been discussed is how 

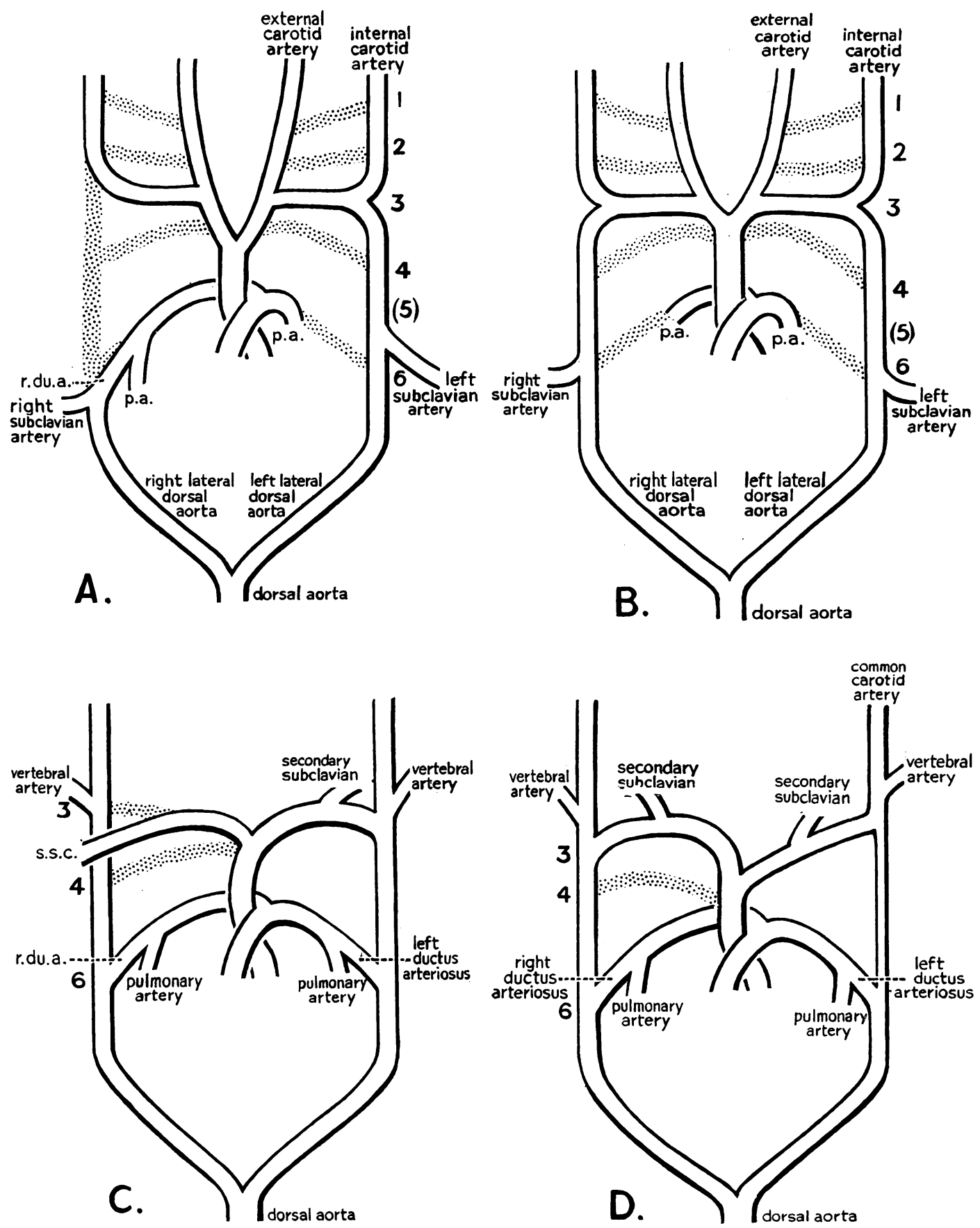

FIG. 2.-Diagrams of ventral views of experimentally produced abnormalities of the arterial arch pattern. (A) and (B) in the rat (modified from Wilson and Warkany) and (C) and (D) in the chick (modified from Stephan). The diagrams have been redrawn for comparison with Fig. 1 .

In (A) there is no systemic arch, and the dorsal aorta is supplied by the left carotid arch and right ductus arteriosus. In (B) there is no systemic arch and the aorta is supplied by both carotid arches and the lateral dorsal aortæ. The fifth arch (5) does not appear during development in the rat. In embryo chicks (C) (ligature of third and fourth right arches) and (D) (ligature of fourth right arch) the dorsal aorta is supplied through the persistent lateral dorsal aortæ joined with the carotid arches and the ducti arteriosi. 
such alterations in blood flow might be established in the first instance by external environmental conditions. In this section it will be necessary to consider the influence of X-irradiation of the fœtus and its nutritional and respiratory requirements.

(a) X-irradiation. The X-irradiation of the fœtus of the albino rat has been the subject of papers by Wilson and his fellow workers. A method was devised by which rat fotuses could be irradiated without irradiation of the mother. By suitable sub-lethal doses of X-rays it was possible to produce considerable malformation without causing the death of the fœtus, although some malformations produced were incompatible with later fœtal life.

It was found that irradiation of $100 \mathrm{r}$ in a single dose caused considerable slowing of fœtal growth for the first day after irradiation, some of which was made up on the second and third days, but a noticeable retardation of growth was found to persist until term. Irradiation on the ninth day of gestation (Wilson et al., 1953) produced some instances of complete situs inversus of the heart and viscera and other specimens showed some degree of reversal of normal symmetry. Many of the abnormalities produced are stated to be similar to those found by Wilson and Warkany (1949) (see below) in instances in which the mother was fed on a vitamin A deficient diet. Heart malformations were defects of the septa and also of the endocardial cushions. Irradiation on the tenth day of gestation (Wilson and Karr, 1951) did not have at all the same striking effects on the heart, but malformations of the aortic arches were produced in that they exhibited some degree of right-sided dominance instead of the left-sided dominance of mammals.

In an analysis of these results, Wilson et al. (1953a) suggested that X-rays might bring about their effects in three ways. Firstly they might cause their effects by killing certain cells, secondly by causing an arrest of mitotic activity, or thirdly by bringing about some subtle genetic changes in the dividing cells.

The difference in degree of susceptibility of the tissues to radiation on the ninth and tenth days is striking. The heart primordia of the rat are first recognizable as paired structures on the tenth day of gestation and by the eleventh day the heart has taken on its typical flexure. Yet, as late as the ninth day it is possible to bring about complete situs inversus. However, by the tenth day a far more stable state has been reached. It seems difficult to believe that, as late as the ninth day, the asymmetry of the fœtus has not been determined. The authors can offer no further explanation of this difficulty.

(b) Nutrition. Wilson and Warkany (1949) produced abnormalities in the arterial system of developing rats by feeding the mothers on a diet very deficient in vitamin A. This materially affected growth. Nevertheless by this means abnormal arterial arch patterns, some very like those produced mechanically by Stephan in chicks, were produced in the embryo rats (Fig. 2, A and B). In the developing rat the stage at which the fourth pair of arterial arches is becoming increasingly important and the first two pairs of arches are disappearing is approximately at the eleventh day of gestation in a total period of twenty-two days. This corresponds to the stage of development of the arches seen in a four-day chick where the period of development is twenty-one days, vascular development of the chick being very precocious. Wilson and Warkany could not influence the appearance of the third arch and the disappearance of the first two pairs of arches; they accounted for this by suggesting that the vitamin A lack only begins to have effect at the eleventh day. The effect of vitamin A lack on the development of the fourth and sixth pairs of arches may be very dramatic, producing very gross anomalies if they do not form properly. The absence of these vessels throws considerable light on the plasticity of the lateral dorsal aortæ for, without going into detail, it may be noted that, if the right lateral dorsal aorta is necessary to keep the circulation going, it will persist. Arch development may also influence septal development, for if the development of the sixth pair of arches is so much interfered with that there is no outlet from the right ventricle, then, as in the chick, the interventricular septum is not completely formed.

Wilson et al. (1953b) followed up those experiments with others in which mother rats were kept on vitamin A deficient diets but massive doses of vitamin A were administered at different stages of pregnancy. So far as arterial arch pattern was concerned, without vitamin A supplement, anomalies occurred in nine per cent of the offspring; administration of large doses prior to the twelfth day of 
gestation prevented all aortic arch anomalies. The arches begin to assume their definitive pattern on the fifteenth day.

So far as the heart was concerned, the anomalies were not entirely prevented by dosing the mother as early as the tenth day. The two most frequently occurring cardiac anomalies were defect of the interventricular septum and defect of the bulbus region. If the mother was dosed on the tenth, eleventh, or twelfth day, bulbar defects were prevented but interventricular septal defects were not, although they occurred less frequently. The authors' remark, "This observation may throw some light on the relationships between the two defects. They occur conjointly with sufficient frequency, both in experimental studies of this sort and in human teratology, to suggest a causal inter-relationship, but it does indicate that the two defects are determined at different times in development."

It is worth noticing, however, that vitamin A deficiency may affect the actual growth of the ventricular septum itself, and ventricular septal defect may thus be produced, not by incomplete fusion of the septal elements but as a result of having so small a septum in the ventricle that it is not large enough to be completed even if the arrangement of the arches and bulbar septum would permit of such completion.

(c) Supply of Gases to the Embryo. It has often been suggested (e.g. Haring, 1956) that congenital cardiac abnormality can be explained either by failure or arrest of growth, in which case fœtal communications remain in the fully formed heart, or by overgrowth by which the normal pathways of the blood streams through and out of the heart tend to become blocked. The idea that such changes in normal development can be brought about by changes in oxygen supply to the tissues has been the subject of experimental work on rats by Haring and Polli (1957). At a suitable stage in gestation the maternal rats were submitted to air with increased carbon dioxide content combined with normal or decreased oxygen tension. In as much as the carbon dioxide present in the blood of the mother will make the tissues more acid than usual, this procedure should result in the release to the tissues of more oxygen than is normal. Thus the fotuses in maternal rats exposed to this increased carbon dioxide content should be hyperoxygenated. Whether this indeed happened is not known, but in the experimental animals hypertrophy of the cardiac muscle was found. This was shown both by the thickening of individual muscle fibres and by the changes in muscle morphology. In some instances overgrowth of the walls of the vessels leaving the heart was also produced. From the illustrations given in the paper referred to it would seem that the experimental evidence of overgrowth, presumably caused by increased oxygen supply to the tissues, is well founded.

The authors go on to deduce that failure of septation may be explained by periods of arrest of growth owing to failure of oxygen supply, and that combined defects might be caused by alternate periods of arrest and overgrowth caused in turn by variations in oxygen supply to the fotal tissues.

\section{Genetic Factors in Heart and Arterial Arch Development}

Evidence of inheritance of congenital heart disease in man has been sought by many authors. Cockayne (1938) has shown that transposition of the viscera can be explained as being the manifestation of an autosomal recessive gene. This gene is somewhat lethal in nature and heart disease is abnormally high in instances of complete transposition of the viscera. The gene does not always produce its full effect and all kinds of gradations from fully normal to highly abnormal conditions of the heart are met with, with more or less complete transposition of the viscera. The most frequent anomaly likely to occur with transposition is Fallot's tetralogy often with some additional anomalies but almost all the known cardiac malformations occur in smaller numbers.

Evidence that heart abnormalities frequently occur in association with arachnodactyly, an abnormality showing dominant autosomal inheritance, has been given by Reynolds (1950). Further evidence of inherited heart disease has been given by McKeown et al. (1953) and by Polani and Campbell (1955).

There is very little known about the inheritance of arch pattern in animals, but the work of Froud (1954) on the fate of the left systemic arch in chicks must be mentioned. In examining chicks that had failed to hatch, he found that 8.8 per cent of such chicks showed persistence of the left systemic 
arch. In day-old chicks he found $7 \cdot 7$ per cent with some trace of the left systemic arch, so that failure of the left systemic arch to close was probably not the cause of death. He was working with fowls of known parentage and concluded that persistence of the left systemic arch was more frequent in the Light Sussex breed of fowl than in the Rhode Island Red.

In Rhode Island Reds he found that the frequency of the anomaly varied in the progeny of individual cocks and hens, and it seems fairly conclusive that for the persistence of the left systemic arch in fowls there is some genetic basis. How the genes bring about the effects just mentioned is quite unknown. If the views of Bremer (1932) as to the importance of the blood streams in keeping open the lumina of the vessels are correct, a possible mechanism is that in those chicks in which both arches persist the growth movements at the preceding stage must be slightly varied so that blood is directed equally into both systemic arches as it leaves the ventricle, whereas normally the blood flowing from the heart is directed into the right systemic arch.

Alternatively, it is possible to imagine that genetic factors influencing the wall of the left systemic arch are normally at work and bring about the closure. In this connection it may be recalled that in mammals the ductus arteriosus closes in two stages: first the sudden cessation of the blood flow brought about by the occlusion of the lumen by the contraction of the walls, and then considerably later the final filling of the channel by endothelial proliferation. For this mechanism to act, genetic factors have prepared the wall of the ductus; it may be that in some analogous manner the wall of the left systemic arch in chicks is normally prepared to close at the appropriate time.

\section{DisCUSSION}

The initial asymmetry of the heart seems to be under genetic control. As development proceeds, it is possible to see how environmental factors, both outside the heart and inside it, come to play a larger part in the details of heart development. Views vary as to how far genetic control of development is continued. In the extreme mechanical view genetic control does not seem to be envisaged after the blood stream has started to move, as, from that time onwards, according to this school of thought, all development follows quite mechanically. The partitioning of the chambers of the heart would seem to be due, basically, to endocardial proliferation, and the closing of arterial vessels such as then became distinct is associated with endothelial growth and with ultimate occlusion, as has been shown by Stephan (1952).

The factors we are seeking are those concerning the differential growth of the lining of the heart and the arteries. The mechanical view would appear to be that in the absence of blood flow endothelial proliferation will take place. The force of the blood stream is therefore the all important factor; from the moment that the blood starts to flow it plays a major part in determining the formation of the heart and its chambers, and the position of the heart and its blood streams in turn determine the development of the great arteries.

The alternative view is one that envisages a prolongation of the time of gene action. For the correct development of any organism or organ it is necessary that the environment, both external and internal, be normal. On this view, the blood flowing through the heart is such a part of the environment, but it is to be looked upon as a part of the environment of the developing heart and not a proven chief determinant. Again on this view, when one of the arterial arches closes, the phenomenon of closure is associated with slowing of blood flow and endothelial proliferation but it does not make either responsible for the other. In the development of the septa this view envisages that proliferation may take place by the operation of a genetic stimulus and so accounts for those instances in which septa grow in the path of blood streams. That mechanical factors, particularly the flow of blood, do have a large part to play in the development of the heart can be seen in some instances when vessels, which might otherwise disappear, are kept open; also when the failure of the interventricular septum to be completed can be ascribed to such mechanical forces.

The development of the interventricular septum may be considered to see how the various factors that have been enumerated may be involved, either separately or together. In order that the :septum shall be completed, the atrium, ventricle, and bulbus must come to lie in the proper relation- 
ship to each other. As Keith (1948) pointed out, the initial twisting of the heart leaves the bulbus too far to the right and in the mammal this extreme position is remedied during later development by changes of proportion of various parts, which Kramer (1942) attributes to differential growth. Foxon (1955) has pointed out that the methods by which this reorientation comes about do not seem to be the same in birds and mammals. However, if this reorientation does not come about correctly, defects of the heart at the level of the bulbus will be produced, such as overriding aorta and pulmonary stenosis. On the face of it, it would seem that such failure of realignment could be brought about either by incorrect action of asymmetrical growth factors, which would give the condition a genetic origin, or by incorrect growth of an environmental nature such as might be produced by vitamin A deficiency or by hyper-oxygenation of the tissues.

These factors may well be termed growth factors and may, for convenience, be divided into $(a)$ incorrect growth, either hypertrophy or gross under-development, or $(b)$ incorrect positioning of related elements. In addition it has to be realized that, even if the growth and position of the various septal elements are correct, then ultimate fusion may be prevented by mechanical effects of flow, such as are found when the blood from the right ventricle cannot pass out through the pulmonary artery which may be missing or very narrow. Thus defects in the ventricle may be secondary to those in the arterial arches. This may be of importance since there is at least some evidence of genetic occurrence of arterial arch irregularities.

It is not easy to seperate genetic and other factors, for growth is affected by hereditary and by environmental factors. Growth of the septa and also the relative growth of the various chambers of the heart may be considered. In the case of the vitamin A deficient rats it would seem that the factors involved are entirely of an external environmental nature, but it is not so easy to maintain that there is no genetic element when some form of differential growth is necessary to realign the chambers of the heart. The internal partitioning of the heart shows a large degree of conformity with flow through it, but here again the interatrial septum seems to appear irrespective of the normal conditions of blood flow and this would indicate that in its formation a powerful genetic factor is at work. The appearance of the partitions in the heart influences blood flow, even if they are not affected by it, and therefore any irregularity in the internal configuration of the heart may have some effect on flow, as Shaner (1949) found in the hearts of pig embryos. It is known that such projections may result from endothelial proliferation and that this may be a result in turn of some infectious agent. For example, one of the effects of virus infection with rubella is endothelial proliferation, and it is possible that this disease may bring about some of its effects by such proliferation either in the heart itself or in the arterial vessels which in turn might have some effect upon the structure of the heart. This may be an example of the normal developmental process being upset by a non-genetic external factor.

We are thus brought up against the main difficulty in assigning a cause to even one form of congenital heart malformation, such as an incomplete ventricular septum. It may be due to a genetic factor, as shown by Cockayne (1938), or it may be caused by the lack of some external factor such as sufficient vitamin A in the maternal diet. The problem that now poses itself is, is it possible to distinguish between incompleted interventricular septa of different ætiology? In as much as the physiological results of the incomplete septum will be the same, a physiological classification of congenital heart disease will be of little help; but a more detailed anatomical consideration might assist. For example, a grossly under-developed interventricular septum might be caused by nutritional deficiency, but a reasonably well developed septum, though incorrectly aligned and so incomplete, might be due to a genetic factor. If these were to be associated with other abnormalities, it might be possible to proceed further. If, for example, the grossly deficient interventricular septum were to be associated with similar failure of the bulbar septa to develop to their normal size, the nutritional basis for the anomaly might be established. If, however, the bulbar septa were present, but divided that structure incorrectly, and were to be associated with an incorrectly placed interventricular septum, a genetic cause could be postulated. This is all highly speculative, but from the evidence that has been brought forward in this article it would seem reasonable to suppose that there 
is more than one cause of congenital heart disease, and even of one apparently similar condition such as Fallot's tetralogy, if that term is used when an incomplete interventricular septum is found with pulmonary stenosis.

If the possibility of more than one cause of such conditions is admitted, it becomes easier to understand the finding of Cockayne (1938) that transposition of the viscera, genetically determined, is frequently accompanied by Fallot's tetralogy, when compared with the finding of Polani and Campbell (1955), who, while showing that there is a genetic basis for this abnormality, also found that there is more chance of a child being born with Fallot's tetralogy if the age of the mother is forty or more. For it is well known that in modern western society, women of this age tend to restrict their diet, often to a marked degree, and it is conceivable-I put it no more strongly than this-that some of the cardiac malformations met with in children of older mothers could be accounted for on a nutritional basis.

Finally, although it is now possible at least to guess at some of the causes of defects of the interventricular septum, of defects of the bulbar region, of abnormalities of the arterial vessels, and of certain of these defects combined, we are as yet without any clear indication of what it is that produces gross defects in the interatrial septum, and there is no doubt from an experimental point of view that this part of the heart requires considerable further study.

\section{SUMMARY}

The embryological development of the heart and arterial arches in the vertebrates is discussed and the relationship between development and phylogeny considered.

The earliest phases of heart development are under genetic control but mechanical factors, particularly the force of the moving blood stream, appear to influence the chain of development materially.

A moving blood stream is necessary for the full development of the heart rudiment, but in amphibians, in some instances, the heart has been found to take on its normal shape without the need of blood flow. Blood flow does not seem to be a necessary stimulus for the appearance of heart septa, but probably the septa are not normally formed unless there is normal blood flow. Ideas vary as to how much of the heart development is governed by genetic factors and how much by mechanical ones.

Evidence obtained by Stephan working on chick embryos and by Wilson and co-workers on rat foetuses suggests that in both animals genetic factors are involved in determining the disappearance of vessels concerned in the circulation of the early embryo: this applies particularly to the arterial arches. Other parts of the arterial arch system, such as the lateral dorsal aortæ seem much more labile and their closure is prevented by the continual passage of blood through them.

The effect of external influences such as X-rays and vitamin supply is considered. X-rays applied at a critical time have an effect on the symmetry of both heart and arterial vessels. This effect is presumably on the system that normally expresses the particular form of symmetry inherited by the individual. The effect of vitamin A deficiency in the fotal rat is on growth. The abnormalities of the heart and arterial arches met with can be explained as variation in normal growth and not in terms of reversal of symmetry.

The growth of various parts of the heart has been shown to be affected by the supply of gas to the embryo.

Several authors have shown genetic factors to be active in heart disease in man. Little is known of the subject in animals but evidence of the inheritance of persistant left systemic arch in domestic poultry has been found.

The likelihood of human congenital heart disease being of hereditary or environmental origin is discussed and it is shown that ventricular septal defects could be due to either cause. It is suggested that even a more complex condition, such as when an incomplete ventricular septum occurs with pulmonary stenosis, might sometimes be genetic and sometimes non-genetic in origin. 


\section{REFERENCES}

Bremer, J. L. (1928). Anat. Rec., 37, 225.

(1932). Amer. J. Anat., 49, 409.

Cockayne, E. A. (1938). Quart. J. Med., 27, 479.

de Beer, G. R. (1951). Embryos and Ancestors. Oxford.

Ekman, G. (1925). Arch. Entwickl. Mech. Org., 106, 320.

Fales, D. E. (1946). J. exp. Zool., 101, 281.

Foxon, G. E. H. (1955). Biol. Rev., 30, 196.

Froud, M. D. (1954). J. Genet., 52, 456.

Garstang, W. (1921). J. Linn. Soc. (Zool.), 35, 81.

Goerttler, K. (1955). Beitr. path. Anat., 116, 33.

Goodrich, E. S. (1916). Proc. roy. Soc. B., 89, 261.

Haring, O. M. (1956). J. int. Coll. Surg., 26, 682. , and Polli, J. F. (1957). Arch. Path., 64, 290.

Huxley, J. S., and de Beer, G. R. (1934). The Elements of Experimental Embryology. Cambridge.

Keith, A. (1948). Human Embryology and Morphology. 6th ed., London.

Kramer, T. V. (1942). Amer. J. Anat., 71, 348.

McKeown, T., MacMahon, B., and Parsons, C. G. (1953). Brit. Heart J., 15, 273.

Pernkopf, E., and Wirtinger, W. (1933). Z. Anat. Entwickl. Gesch., 100, 563.

Polani, P. E., and Campbell, M. (1955). Ann. hum. Genet., 19, 209.

Reynolds, G. (1950). Guy's Hosp. Rep., 99, 178.

Rudnick, D. (1944). Quart. Rev. Biol., 19, 189.

Shaner, R. F. (1949). Amer. J. Anat., 84, 431.

Simons, J. R. (1957). J. Embryol. exp. Morph., 5, 250.

Stephan, F. (1952). Bull. biol., 86, 217.

Wilson, and Warkany, J. (1949). Amer. J. Anat., 85, 113.

- , and Karr, J. W. (1951). Amer. J. Anat., 88, 1.

_, J. G., Jordan H. C., and Brent, R. L. (1953a). Amer. J. Anat., 92, 153.

-, Roth, C. B., and Warkany, J. (1953b). Amer. J. Anat., 92, 189. 